\title{
DANIELI UNIVERSAL ENDLESS (DUE) - THE NEW EVOLUTION OF DANIELI THIN SLAB CASTING AND ROLLING PLANT*
}

\author{
Alessandro Pigani ${ }^{1}$ \\ Paolo Bobig ${ }^{2}$ \\ Mike Knights ${ }^{3}$ \\ Stefano Martinis ${ }^{4}$
}

\begin{abstract}
Over the last 25 years the thin slab casting and rolling process has been gaining a major market share in the production of hot rolled strip, progressively eroding the areas previously of exclusive domain of conventional mills. This is mainly thanks to the competitiveness of the process over conventional ones, and to the growing ability of this technology to cover the majority of market niches, well beyond the boundaries of the commodity market. After having significantly contributed to the continuous expansion of this production mode and after an extensive campaign of theoretical research and physical testing in reference plants, Danieli has progressively developed over the years a new generation of thin slab casting and rolling layout, marking a new phase in the evolution of this process. The new step-ahead is epitomized by the name QSP-DUE. This configuration belongs to the Quality Strip Production (QSP) family, which is Danieli's strip-quality-oriented thin slab based plants. The innovative features of the process are embodied in the acronym DUE, which stands for Danieli Universal Endless (Danieli patented technology - US patent n. 8087449, January 3rd, 2012). What makes this new concept different is the ability of "universal" rolling modes thanks to the fact that, now, within one single plant it is possible to perform coil-to-coil, semi-endless and endless rolling modes, making this plant a major technology innovation compared to the previous generation.
\end{abstract}

Keywords: QSP-DUE; Flexibility; Endless; Ultra-thin gauges.

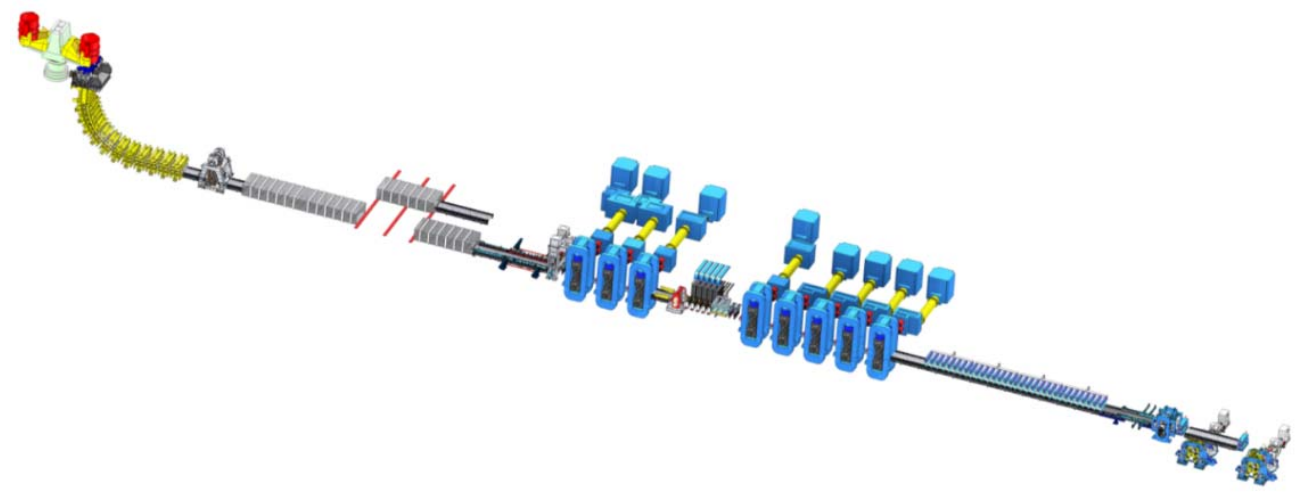

Product Manager, Danieli Wean United, Italy.

Director Process Technology, Danieli Wean United, Italy.

Executive Vice President Sales, Danieli Davy Distington, Italy.

Executive Vice President B.U. Flats, Danieli Automation, Italy. 


\section{INTRODUCTION}

This new approach can be seen as the natural evolution of the original, successful Danieli concepts that marked the progress of the thin slab route, namely the application of the vertical curved design thin slab caster and the separation of the rolling stands into high reduction units and finishing units.

Since the beginning they have represented the fingerprints of Danieli QSP technology and have become a source of inspiration for most of the recent innovations in the industry, confirming the advantages of Danieli's original concept.

At present, two different layout concepts have been developed for the thin slab route:

$>$ Plants conceived with 1 or 2 casting strands, connected to the rolling mill through long tunnel furnace(s), which have the function of reheating and equalizing slab temperature as well as of guaranteeing sufficient buffer time in case of either scheduled stoppages of the mill (i.e. work rolls change) or unscheduled interruptions of material flow. Danieli consolidates this QSP layout (fig. 1) by reaching world record results, both in productivity and quality.

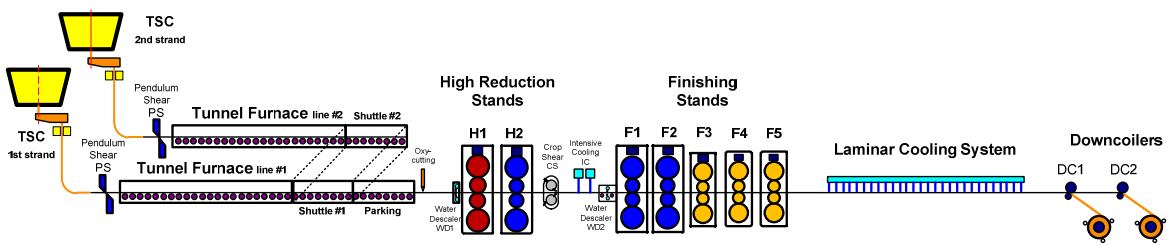

Figure 1: Quality Strip Production (QSP) configuration

$>$ In recent years, thanks to a progressive increase in mass flow due to increased casting speeds, inherited from the last generation of thin slab casters operating at ultra-high speeds, in combination with the reliable introduction of induction heating technology in place of tunnel furnace, it was possible to develop the QSP-E layout (fig. 2): a new generation of extremely compact plants, specifically dedicated to the production of ultra-thin gauges. The latter made possible thanks to the application of the endless rolling process (i.e. the direct and uninterrupted connection between casting and rolling), to overcome the obstacles represented by the well-known problems of strip threading when producing thin gauges in coil-to-coil mode.

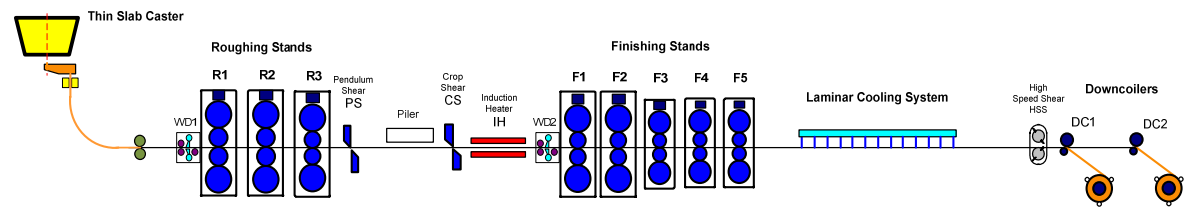

Figure 2: Quality Strip Production Endless (QSP-E) configuration

The QSP plants have made it possible to drastically widen the steel grades mix of thin slab based processes and to ramp up plant productivity well in excess of 3 Mtpy, also thanks to the possibility of operating with 2 casting strands. These plants were initially conceived for the application of coil-to-coil rolling and then for the semiendless process to roll thinner gauges below $1 \mathrm{~mm}$. On the other hand, the QSP-E concept made it possible to optimize the production of ultra-thin gauges in endless mode, while showing little flexibility in the production of more sophisticated grades, due to the rigid link between casting and rolling. In case of endless rolling, the caster is in fact forced to always run at very high casting speeds and this is not possible for all the steel grades due to metallurgical reasons. Moreover, the endless process has 
proven to be economically competitive only for the production of coils having strip thickness below $1.5 \mathrm{~mm}$, but as soon as the strip thickness is increased, the power required by the induction heaters to continue operating in endless mode becomes excessively high, making it economically necessary to return to coil-to-coil mode. Since the QSP-E has a compact layout configuration, with slab re-heating provided by the induction heating system, there is no buffer time between caster and mill and this creates significant limitations on the operational flexibility of the entire production line. Additionally, plant yield and productivity are affected by the absence of buffer because caster restranding has to be synchronized with roll changes in the mill, which are quite frequent in endless mode, due to significant wear and thermal deterioration of the rolls in continuous contact with the strip. As a consequence, caster sequencing (i.e. plant yield optimization) is reduced compared to plants operating in coil-to-coil mode, where the sequence length is determined by SEN refractory life and not by roll change scheduling. Also, the QSP-E layout does not allow the plant's capacity to be doubled by adding a second casting strand, hence limiting the overall productivity in the range of $2 \mathrm{Mtpy}$, depending on the product mix. This in spite of significant investment cost, thus making the specific investment per ton not particularly attractive. All in all, these factors have negatively influenced the expansion of the endless process, notwithstanding its indisputable advantages, when it comes to ultra-thin gauge production.

\section{THE DANIELI UNIVERSAL ENDLESS (DUE) CONCEPT}

In a continuous effort to improve existing processes and technologies and overcome their current limitations, Danieli has developed a new concept in thin slab casting and rolling plants, able to unify in a single production line all the winning features that up to now have been developed using different approaches, while eliminating the limiting factors of each one of them.

This is the backbone concept behind the development of the Danieli Universal Endless layout, which features the following:

$>$ High productivity, thanks to an unprecedented combination of slab thickness and speed.

$>$ High production flexibility, able to operate in coil-to-coil, semi-endless and endless rolling mode.

$>$ High operational flexibility, due to the presence of the tunnel furnace and relevant buffer time.

$>$ It covers the full spectrum of steel grades produced for flat products, including the most sophisticated ones rolled via thermo-mechanical rolling (API pipeline grades) or temperature-controlled rolling (multiphase products) as well as the grades which require moderate casting speeds like peritectic, electrical steels and high-carbon grades, being crack-sensitive.

$>$ It covers the full spectrum of geometrical strip dimensions, ranging from $0.8 \mathrm{~mm}$ ultra-thin gauges, produced in endless mode, up to $25 \mathrm{~mm}$ thick strips. This, of course, in combination with an unbeatable transformation cost, lower than any other process presently available.

\subsection{Thin Slab Caster}

The DUE vertical-curved caster features 5.5-m main radius, designed to operate with a single slab thickness of $110 \mathrm{~mm}$ after dynamic soft reduction, within the casting 
speed range from 2.5 up to $6.8 \mathrm{~m} / \mathrm{min}$, depending on the steel grade. Such an unprecedented combination of slab thickness and speed (fig. 3) makes it possible to easily reach the mass flow conditions required by the full endless production mode, particularly when ultra-thin gauges of commercial grades are produced. This caster, the logical development of the already consolidated vertical curved Danieli design, takes full advantage of the experience acquired over more than 25 years dedicated to perfecting this design, as well as the considerable experience gained in ultra-high speed casting. Industrial operation at high speed has been extensively experienced since 2008 in Posco CEM minimill (Korea), where Danieli thin slab caster usually works in the speed range from 6.0 to $7.0 \mathrm{~m} / \mathrm{min}$ (fig. 4), with peak of $8.0 \mathrm{~m} / \mathrm{min}$.

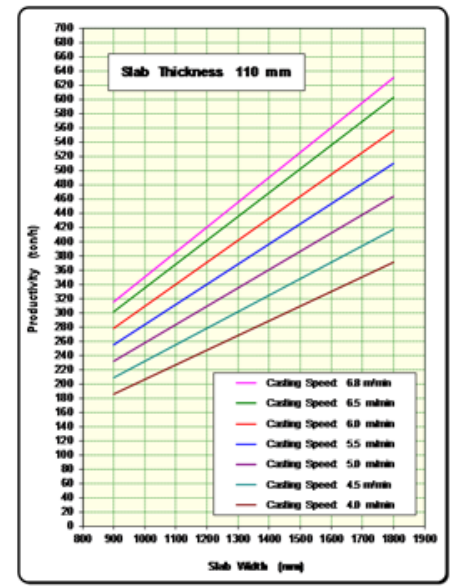

Figure 3 (Left): Danieli Universal Endless (DUE) - caster productivity vs. slab width

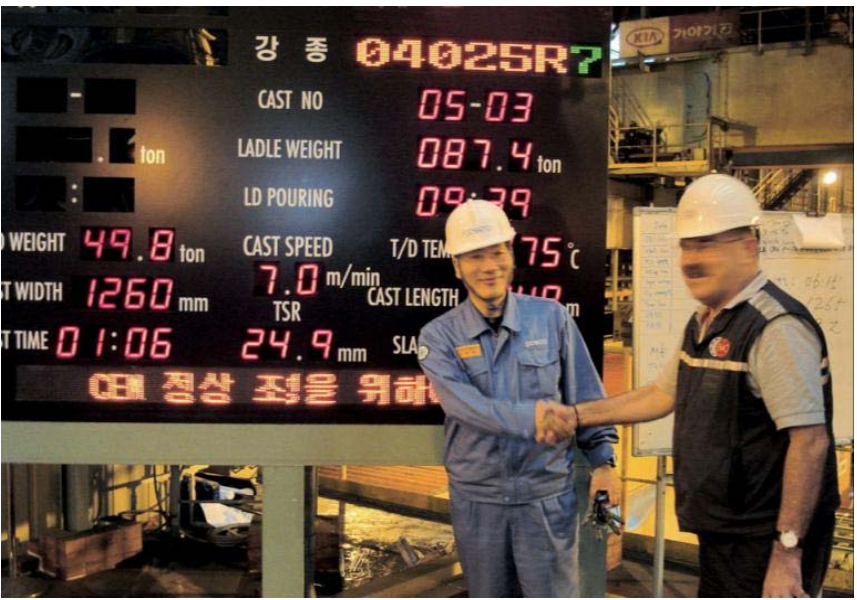

Figure 4 (Rigth): Snapshot from Posco CEM minimill (Korea) during commissioning period

Special patented mould and oscillator design, dynamic air-mist secondary cooling, plus dynamic soft reduction ensure superior slab quality. The combination of the afore mentioned slab geometry and caster design features make it possible to obtain a mould fluid-dynamic similar to a medium slab, resulting in a higher control of the solidification process and in a much easier and more stable operation compared to a conventional thin slab process. Stability and easiness of operation are key points for endless operation, where repeatability and steadiness of operation are a must. Danieli, since the beginning of the nineties, has been the first company to develop and then utilize in an industrial way the dynamic soft reduction as basic tool in all its thin slab casters, in conjunction with the vertical curved design which provides the best control of internal and surface slab quality in any casting conditions. It provides 
the mill with a thicker slab compared to the traditional thin slab approach, with a remarkable increment in the reduction ratio from slab to strip for this kind of plant, which in turn allows the production of a wider product mix. The possibility of covering the entire production range with one slab thickness only, regardless of final product gauge, clearly brings significant operating advantages. The caster can therefore produce up to about 3 Mtpy on a single casting strand, covering a wide range of steel grades such as: low, medium and high carbon, high strength low alloyed, peritectic, silicon, pipeline, API and more. The flexibility of Danieli's mould design, which guarantees stress/strain-free solidification in the shell, makes it possible to perform continuous slab width adjustment during casting. The absence of limitations or constraints in the slab width makes production flexible and profitable.

\subsection{Tunnel Furnace}

The tunnel furnace is conceived to operate in coil-to-coil mode like in conventional thin slab rolling plants, in semi-endless mode, able to accommodate long mother slabs that are the equivalent of several coils, and in full endless mode.

This unit provides a fundamental buffer function that increases plant flexibility and provides the possibility of performing work roll changing as a background task, without affecting the

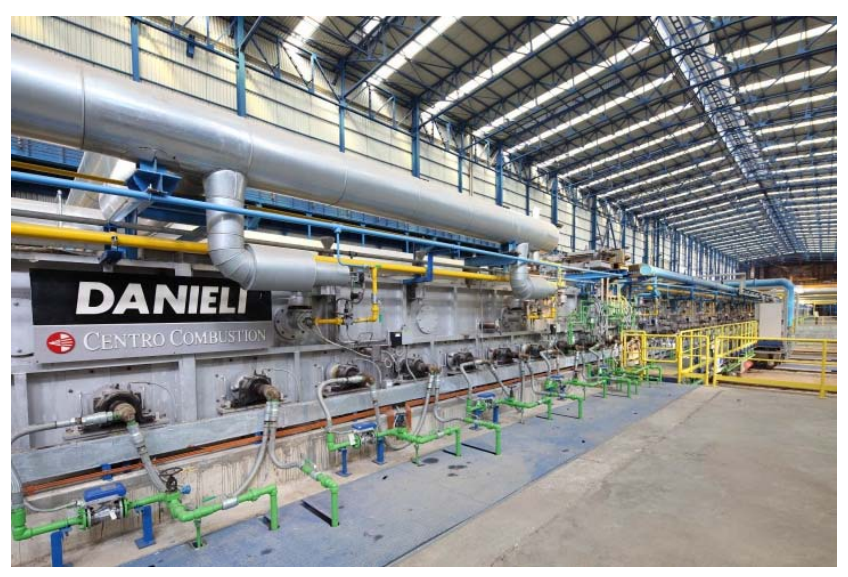
operation of caster and meltshop, "switching" from endless to coil-to-coil operating mode during roll changes, which in any case does not reduce plant productivity.

\subsection{Rolling Mill}

The DUE mill is the natural evolution of Danieli's QSP concept, which features the well-known and already successfully proven configuration with separation of the mill stands into high reduction units and finishing units (fig. 6), in order to perform dual step rolling.

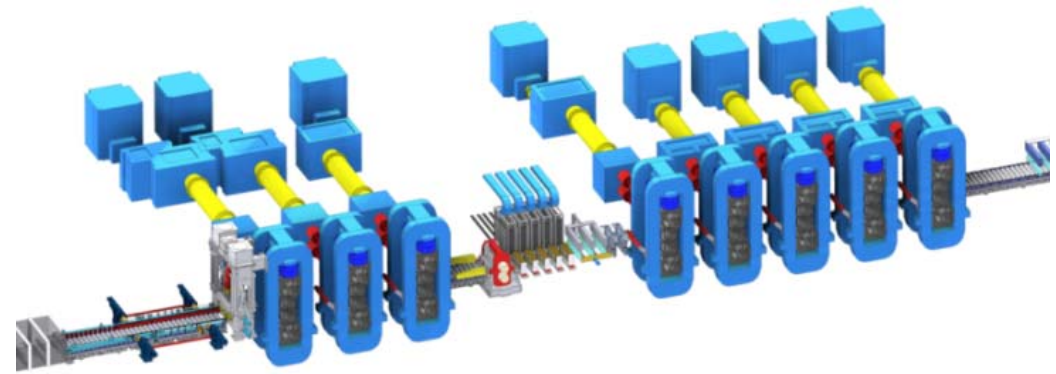

Figure 6: Rolling mill area configuration

This configuration includes:

$>$ A dedicated high-pressure descaling unit at finishing mill entry (in addition to the one at high reduction stand entry), to limit imprinting scale phenomena on the bar, thus significantly improving the surface quality of the final coil; 
$>$ An intensive cooling system, incorporated into the descaler box at finishing mill entry, used when producing TMR and/or API grades in order to guarantee the correct bar temperature profile and proper control of grain growth that are essential features of the thermo-mechanical rolling process;

$>$ A crop shear, beneficial for thin gauge production, used to cut the transfer bar head and/or tail end, in order to have smoother threading into the finishing mill as well as reducing tail chew-up;

$>$ An induction heating system, for consistent production of thin and ultra-thin gauges in endless mode. Induction heaters are individually mounted on a retractable frame so they can be transferred off-line for maintenance purposes or when they are not being used (i.e. during coil-to-coil production mode).

The number and size of mill stands are designed to cover a wide range of strip thickness, from $25 \mathrm{~mm}$ down to $0.8 \mathrm{~mm}$, starting from 110-mm-thick slabs, guaranteeing superb geometrical and mechanical properties.

The mill stands are equipped with all state-of-the-art features for utmost strip crown and profile control, bearing in mind that these control functions also have to be operational under load conditions as required by the endless rolling process.

The line is complete with laminar cooling system and coiling area, made up of a highspeed shear (used to cut the coil to length when working in endless or semi-endless mode), threading devices, pinch rolls and downcoilers, already fully tested and presently in operation at the Arvedi plant, Italy (fig. 7).

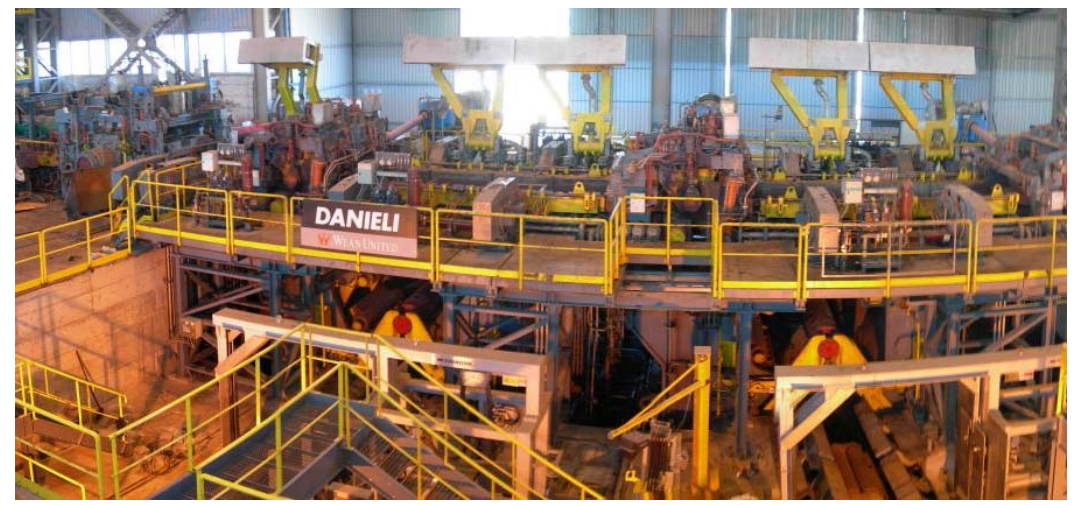

Figure 7: High-speed shear and high-speed downcoiler area

\subsection{Automation}

Using the original technology developed by Danieli Automation, named $3 Q$, it was possible to overcome the challenges the DUE process posed for the automation system. In fact, not only is the automation system required to control the process but it also has to assist operators in properly running the process, providing the necessary experience to develop an innovative process (fig. 8).

Since DUE is a highly integrated process, encompassing casting, reheating, rolling, cooling and coiling, a single virtual pulpit solution was selected. This means that all operators can easily communicate either verbally or visually, consolidating the concept of "one crew" running the plant. Moreover the operators are assisted in managing the plant by a new operator interface concept covering multiple hierarchical levels, each one of which oriented to a specific operational function. 
Changing the strip thickness during rolling requires a very accurate tracking system and a set of smart strategies to simultaneously achieve the required thickness variation while minimizing the transition length. The same concept applies to width changing during casting. Both technologies strongly improve the plant's utilization factor, adapting "in-line" production to orders, and reducing the number of coil widths kept in inventory.

To obtain the required mechanical properties of the rolled strip in all operating modes, the transfer bar and strip cooling are controlled by an integrated set of sophisticated cooling models that combine physical equations with an advanced adaptation system.

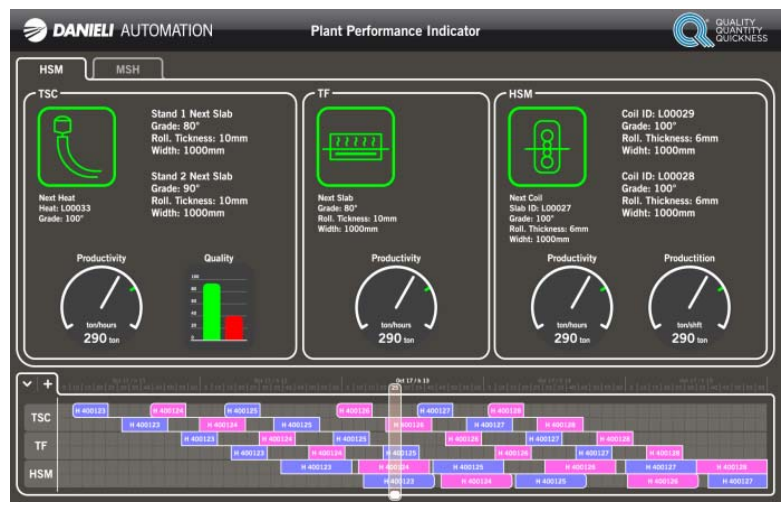

Figure 8: Danieli Automation (DA) 3Q technology

Synchronizing caster and rolling mill is one of the enabling technologies for endless rolling. A new control algorithm has been developed to provide a smooth, reliable link between caster and rolling mill in all operating conditions, while continuing to produce top quality strip. Temperature control using induction heaters provides increased heating efficiency. The new unique design is based on a fully digital control of the heating power and a smart circuit diagram that have made it possible to obtain high heating efficiency while simplifying power pack serviceability. Induction heaters are completely designed and manufactured in-house, on the basis of a high quality control plan to achieve uniform machine ratings. Crown, flatness and wear control in endless plants is paramount. Danieli has developed a complete set of on-line mathematical models to achieve the target crown, flatness and edge drop on the final strip. Control strategies are changed "on-the-fly" according to the actual rolling mode, the expected rolling campaign and in order to use the most effective actuator in each section of the plant.

\section{ROLLING MODES}

As mentioned above, thanks to its layout configuration, the Danieli Universal Endless is able to work in coil-to-coil, semi-endless and endless rolling modes, selecting the most suitable production mode according to final product specification (i.e. endless mode is economically convenient for strip thickness $\leq 1.5 \mathrm{~mm}$ ).

\subsection{Coil-to-Coil Rolling Mode}

The slab generated in the caster is cut to length by the pendulum shear at caster exit: each slab generates a single coil. 
In coil-to-coil production mode caster and mill operate with different mass flows; each one can be optimized according to the specific needs of each unit, which are different, in order to guarantee the best product quality.

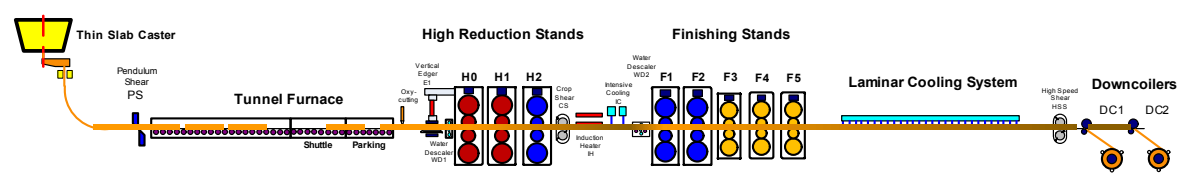

Coil-to-coil process is advisable for the production of strip thicknesses $\geq 1.4 \mathrm{~mm}$. However, applying an intermediate bar reheating with the induction heating system it is possible to have a more stable and reliable rolling condition for the production of thin gauges down to $1 \mathrm{~mm}$ (fig. 9), without having to speed up the bar, once the coiling operation starts, in order to limit temperature losses.

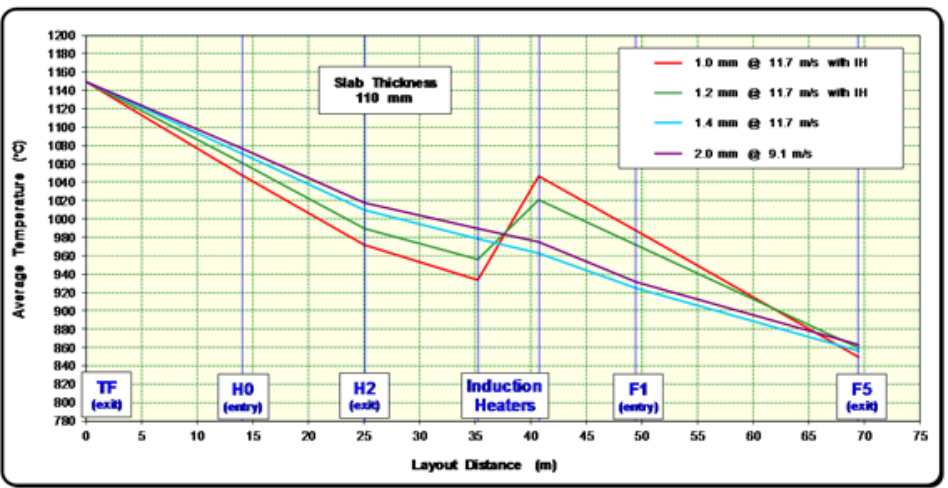

Figure 9: Coil-to-coil rolling - temperature pattern vs. strip thickness

\subsection{Semi-Endless Rolling Mode}

The mother slab generated in the caster (corresponds in length to approx. five coils) is cut to length by the pendulum shear at caster exit: after rolling coils are cut to length by the high speed shear at coiling area entry.

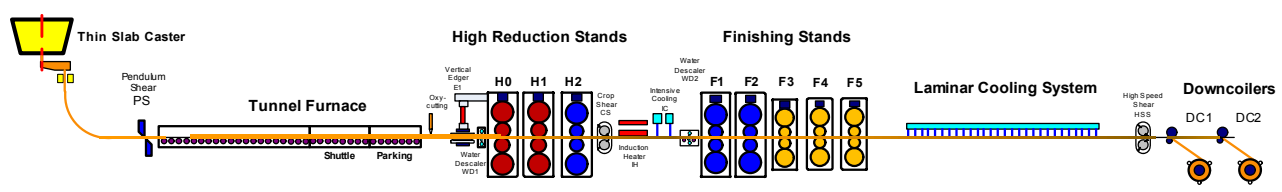

In semi-endless production mode caster and mill operate with different mass flows, just like coil-to-coil production mode. However, in this case there is less material loss, since there are less head and tail end crops at the crop shear at finishing mill entry, due to the smaller number of slabs being threaded into the mill.

Furthermore, it is possible to reduce the minimum strip thickness rolled in the mill, since for the coil generated from the central portion of the mother slab, even when the rolling speed is increased, there is no risk of "flying head" on the run out table, as the strip is already under tension between mill stands and downcoiler.

In the DUE layout the semi-endless process is mainly applied for the production of thin strips if the endless process cannot be applied due to insufficient mass flow, i.e. in the case of MC HSLA or HC grades, or in the case of liquid steel shortages. 


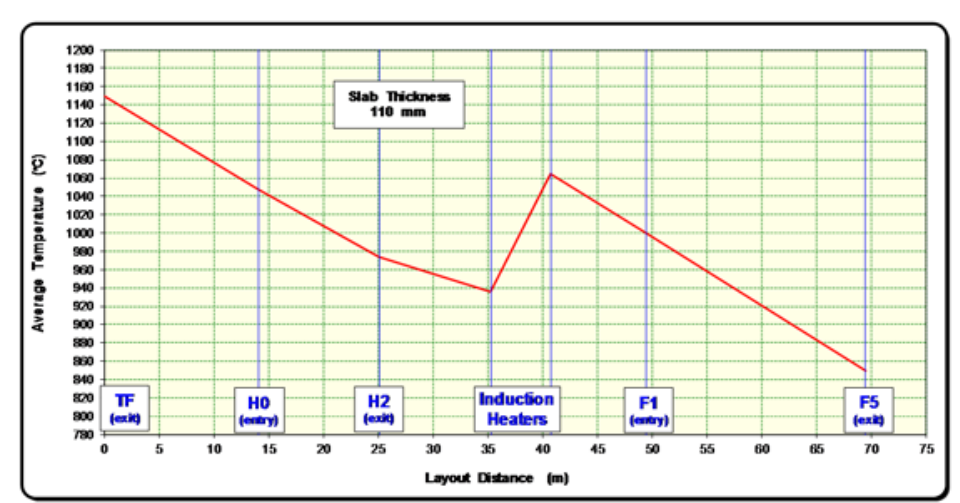

Figure 10: Semi-endless rolling - temperature pattern for 0.8-1.0 mm strip

With the DUE configuration, even during production in semi-endless rolling mode, it is possible to have a more stable and reliable rolling condition for the production of thin gauges, thanks to the induction heating system, without having to speed up the bar when the coiling operation starts, in order to limit temperature losses (fig. 10).

Please note that without the semi-endless process, the DUE layout can be even more compact, since for coil-to-coil and endless mode, the length of the tunnel furnace alone can be shortened to less than $100 \mathrm{~m}$, thus further reducing Capex and Opex.

\subsection{Endless Rolling Mode}

Recent developments in ultra-high casting speed pioneered by Danieli mean that the thin slab caster can now generate sufficient mass flow for a full endless process. The slab generated in the caster is continuously rolled in the mill in endless mode: coils are cut to length by the high speed shear at coiling area entry.

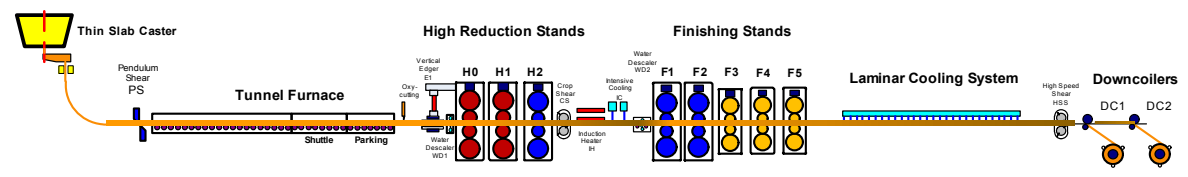

In endless production mode caster and mill operate with the same mass flow in order to ensure an uninterrupted process. With the DUE layout the endless rolling mode is mainly utilized for massive production of thin and ultra-thin strips, overcoming the difficulties implied by other production mode as well as with higher plant yield.

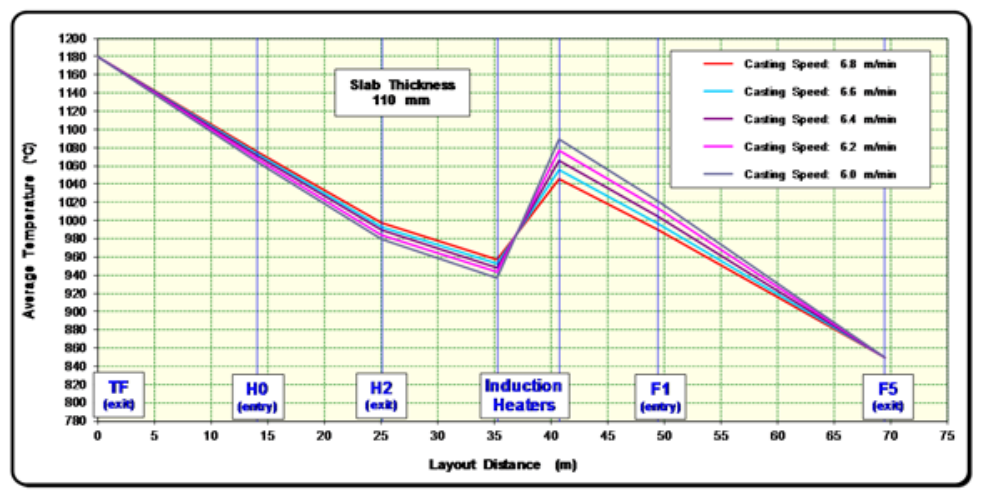

Figure 11: Endless rolling - temperature pattern vs. casting speed 
With the DUE configuration, thanks to the proper mass flow of a 110-mm-thick slab at a casting speed in excess of $6 \mathrm{~m} / \mathrm{min}$, the induction heating system allow stable production of ultra-thin gauges down to $0.8 \mathrm{~mm}$, in stable and repetitive conditions, with strip temperature at finishing mill exit in the area of $850^{\circ} \mathrm{C}$, regardless of the casting speed (fig. 11).

\subsection{Thermo-Mechanical Rolling Mode}

The installation of an intensive cooling system between the high reduction stands and finishing mill stands allows production of high added value pipeline grades such as API. The intensive cooling system is applied in coil-to-coil mode and, when in use, the induction heating system is put off-line. It is an essential tool for applying a real thermo-mechanical rolling process as it cools the transfer bar below the norecrystallization temperature (fig. 12).

In this way the risk of partial recrystallization in the finishing mill and the consequent non-uniform microstructure is prevented, and the coils produced are also suitable for arctic applications (down to $-60^{\circ} \mathrm{C}$ ).

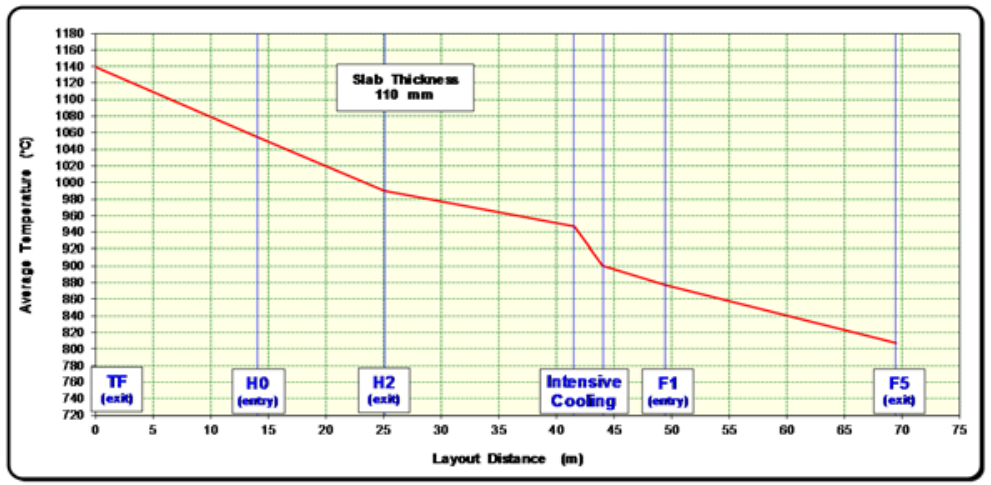

Figure 12: Coil-to-coil thermo-mechanical rolling - temperature pattern for $12 \mathrm{~mm} \mathrm{API} \mathrm{X70}$

With a 110-mm-thick starting slab, the reduction ratio that can be obtained with the DUE configuration from slab to transfer bar and from transfer bar to the final strip can be higher than in other thin slab route plants, allowing the production of API grades in greater thicknesses and making it possible to apply spiral welded pipes in highpressure large diameter gas transportation lines. Additionally, the use of a powered cooling system on the run-out table allows further grain refinement and transformation hardening, taking advantage of the potential of acicular ferrite and bainitic microstructures. In this way it is possible to save on the cost of expensive alloying additions and increasing the strength of the produced coils.

\section{CONCLUSIONS}

The DUE (Danieli Universal Endless) is Danieli's solution to the existing limitations of current thin slab casting and rolling configurations, and constitutes a universal tool able to reach all the production niches of the flat products market, by applying the process that is best suited to each steel grade and product.

Thanks to the combination of slab thickness and casting speed, never-before-seen production rates are possible, up to 3 Mtpy on a single casting strand, making the Capex investment per ton extremely attractive. 
The layout configuration, together with an effective combination of thermal energy (gas-fired tunnel furnace) and induction heating, optimizes energy consumption, making DUE a truly "green" plant, while also reducing Opex by about 15-20 \% compared to the current benchmark of thin slab-based plants.

Coil-to-coil and endless, thermo-mechanical and multi-phase, ultra-thin and thick products are now all possible in a single production line.

This makes both niche products with high-added value and the mass production commodities segment within reach of this new plant, which today represents the most extensive application of Danieli's concept of flexibility. 